\title{
Voxel Orientation
}

National Cancer Institute

\section{Source}

National Cancer Institute. Voxel Orientation. NCI Thesaurus. Code C156500.

The orientation of the voxel relative to the coordinate system of the scanner. May be measured in degrees or radians depending on the scanner make and model. 